\title{
Determining the stability and activation energy of Si acceptors in AlGaAs using quantum interference in an open hole quantum dot.
}

\author{
D.J. Carrad,${ }^{1}$ A.M. Burke, ${ }^{1}$ O. Klochan,${ }^{1}$ A.M. See, ${ }^{1}$ A.R. Hamilton, ${ }^{1}$ \\ A. Rai, ${ }^{2}$ D. Reuter, ${ }^{2}$ A.D. Wieck, ${ }^{2}$ and A.P. Micolich ${ }^{1, *}$ \\ ${ }^{1}$ School of Physics, University of New South Wales, Sydney NSW 2052, Australia \\ ${ }^{2}$ Angewandte Festkörperphysik, Ruhr-Universität Bochum, D-44780 Bochum, Germany
}

(Dated: July 17, 2018)

\begin{abstract}
We fabricated an etched hole quantum dot in a Si-doped (311)A AlGaAs/GaAs heterostructure to study disorder effects via magnetoconductance fluctuations (MCF) at millikelvin temperatures. Recent experiments in electron quantum dots have shown that the MCF is sensitive to the disorder potential created by remote ionised impurities. We utilize this to study the temporal/thermal stability of $\mathrm{Si}$ acceptors in $p$-type AlGaAs/GaAs heterostructures. In particular, we use a surface gate to cause charge migration between $\mathrm{Si}$ acceptor sites at $T=40 \mathrm{mK}$, and detect the ensuing changes in the disorder potential using the $\mathrm{MCF}$. We show that $\mathrm{Si}$ acceptors are metastable at $T=40 \mathrm{mK}$ and that raising the device to a temperature $T=4.2 \mathrm{~K}$ and returning to $T=40 \mathrm{mK}$ is sufficient to produce complete decorrelation of the MCF. The same decorrelation occurs at $T \sim$ $165 \mathrm{~K}$ for electron quantum dots; by comparing with the known trap energy for Si DX centers, we estimate that the shallow acceptor traps in our heterostructures have an activation energy $E_{A} \sim$ $3 \mathrm{meV}$. Our method can be used to study charge noise and dopant stability towards optimization of semiconductor materials and devices.
\end{abstract}

PACS numbers: 73.23.-b, 72.20.-i, 73.63.Kv

\section{INTRODUCTION}

A significant outcome of device miniaturization is the semiconductor quantum dot - a device where electrons or holes are confined in all three spatial dimensions at a length scale comparable to the electron or hole wavelength. These can be made in any semiconductor, in principle, but the $n$-type AlGaAs/GaAs heterostructure is predominant for electrical studies because of the high electron mobilities that can be obtained through growth by molecular beam epitaxy (MBE) 1.2 Small AlGaAs/GaAs dots isolated by tunnel barriers from source and drain electron reservoirs were used as 'artificial atoms $\underline{\underline{3}}$ for fundamental studies of the physics of few-electron systems, $\stackrel{4}{\underline{4}}$ and now play a vital role in the study of electron spin physics ${ }^{5}$ and the early development of quantum computation architectures ${ }^{6}$ Larger AlGaAs/GaAs dots with stronger coupling to the reservoirs have been central to studies of mesoscopic conduction, quantum interference phenomena and electron transport in the quasi-ballistic and ballistic limits, where an electron's mean-free path is comparable to or exceeds the dot's confinement width. ${ }^{7-9}$

A hallmark of these larger 'open' dots is the appearance of fluctuations in the magnetoconductance $G(B)$ at low temperature $T<1 \mathrm{~K}$, which arise from the Aharonov-Bohm effect 10,11 These fluctuations are reproducible for a given dot on a single measurement cooldown, 11 providing a 'magnetofingerprint' of the electron trajectories within the dot ${ }^{12}$ Recent experiments 13,14 highlight the important role that the dot geometry, and the remote ionized dopant potential it contains, play in determining these trajectories, even in the ballistic limit. For a single electron dot with fixed geometry, the mag- netoconductance fluctuations (MCF) change markedly upon raising the temperature to $T \sim 165 \mathrm{~K}$ briefly and returning to low $T$ to remeasure $G(B)$ for a modulationdoped AlGaAs/GaAs heterostructure $\stackrel{14}{=}$ However, for an undoped dot, the MCF remain identical under thermal cycling to temperatures as high as $300 \mathrm{~K} \stackrel{13}{\underline{13}}$ The change in MCF of the modulation-doped electron dot arises from the spontaneous re-organization of trapped charge in the Si doping layer at $T \geqslant 165 \mathrm{~K}$, which alters the dot's potential landscape. The sensitivity of the MCF to smallangle Coulomb scattering by remote ionized dopants provides a potential new application for semiconductor quantum dots as sensitive detectors of fluctuations in dopant ionization state $\stackrel{13}{\underline{13}}$ This could be combined with other methods if needed, e.g., switching noise measurements in quantum point contacts (QPCs) $\stackrel{15}{\stackrel{15}{1}}$ to enable studies of how dopants influence the electronic properties of a given device, as we do here, or for optimizing materials growth to obtain devices with high stability and low operating noise. $\frac{15.16}{}$

Here we demonstrate the potential for investigating the temporal and thermal stability of $\mathrm{Si}$ acceptors in a (311)A-oriented Si-doped AlGaAs/GaAs heterostructure using measurements of the MCF in a hole open quantum dot etched into the heterostructure. We do so by utilizing the measured MCF as a magnetofingerprint of the disorder potential generated by a given spatial charge configuration in the partially-ionized acceptor layer. (311)A-oriented Si-doped AlGaAs/GaAs heterostructures are a key materials platform for studying the fundamental physics of low-dimensional hole systems, where the much stronger spin-orbit interaction obtained via the GaAs valence band's p-orbital-like nature gives rise to interesting topological phase 17 and spin anisotropy 
18-22 effects.

A significant problem with (311)A-oriented Sidoped AlGaAs/GaAs heterostructures is the hysteresis/instability that arises when using a surface metal 'gate' electrode to electrostatically alter the density of the two-dimensional hole gas (2DHG) formed at a buried AlGaAs/GaAs interface within the heterostructure. ${ }^{23-25}$ We recently reported that this hysteresis contains two contributions at different time/energy scales: a) surfacestate trapping at the gate-heterostructure interface, and b) fluctuations in ionization state of $\mathrm{Si}$ dopants. ${ }^{26,27}$ We suggested acceptors dominate the gate hysteresis at $T<4 \mathrm{~K}$ by comparison with higher temperature data in $n$-type (100)-oriented Si-doped AlGaAs/GaAs heterostructures where the surface-state contribution to gate hysteresis is negligible and donor fluctuations cause gate hysteresis. ${ }^{26}$ The hole quantum dot data we present here confirms the low $T$ behavior observed for $p$-type devices in Ref ${ }^{26}$. We show that some fraction of the Si acceptors in $\mathrm{Al}_{0.34} \mathrm{Ga}_{0.66} \mathrm{As}$ act as very shallow trap sites that remain metastable, even at $T=40 \mathrm{mK}$. This is in stark contrast to $\mathrm{Si}$ donors in $\mathrm{Al}_{0.34} \mathrm{Ga}_{0.66} \mathrm{As}$ where the activation temperature is much greater due to the formation of DX centers,$\stackrel{28,29}{2}$ which are donors that can also act as deep, long-lived traps. This capacity allows DX centers to trap charge released by the much shallower ordinary dopants, and gives nanoscale electron quantum devices their high stability at low $T$.

\section{METHODS}

The hole quantum dot was fabricated from a $p$-type modulation doped $\mathrm{Al}_{0.34} \mathrm{Ga}_{0.66} \mathrm{As} / \mathrm{GaAs}$ heterostructure grown on half of a 2" GaAs (311)A semi-insulating substrate by molecular beam epitaxy (MBE). First, a $50 \mathrm{~nm}$ undoped GaAs layer was grown, followed by an undoped, 20-period superlattice with a period of $2 \mathrm{~nm} \mathrm{GaAs}+$ $2 \mathrm{~nm} \mathrm{AlAs} \mathrm{to} \mathrm{trap} \mathrm{eventual} \mathrm{segregating} \mathrm{impurities} \mathrm{at} \mathrm{its}$ interfaces. The active region was then grown, consisting of $650 \mathrm{~nm}$ undoped GaAs, $35 \mathrm{~nm}$ undoped $\mathrm{Al}_{0.34} \mathrm{Ga}_{0.66} \mathrm{As}$ spacer, a homogeneously Si-doped $80 \mathrm{~nm} \mathrm{Al}_{0.34} \mathrm{Ga}_{0.66} \mathrm{As}$ layer and a $5 \mathrm{~nm}$ undoped GaAs cap to prevent oxidation of the Al-content. The Si-doped layer $\left(N_{S i}=\right.$ $3.5 \times 10^{16} \mathrm{~cm}^{-3}$ ) provides charge carriers to a highmobility 2DHG located on the GaAs side of the interface between the undoped $\mathrm{Al}_{0.34} \mathrm{Ga}_{0.66} \mathrm{As}$ layer and the $650 \mathrm{~nm}$ GaAs layer. Si was used due to its low diffusion rate compared to $\mathrm{Be}$, and its amphoteric nature, which yields predominantly $n$-type incorporation on the (100)-surface, and $p$-type incorporation on the (311)Asurface ${ }^{30}$ At room temperature in the dark, we measured a hole density $p=2.22 \times 10^{12} \mathrm{~cm}^{-2}$ and mobility $\mu=141 \mathrm{~cm}^{2} \mathrm{~V}^{-1} \mathrm{~s}^{-1}$. At $T=4.2 \mathrm{~K}$ we obtained $p=$ $1.63 \times 10^{11} \mathrm{~cm}^{-2}$ and $\mu=1.03 \times 10^{6} \mathrm{~cm}^{2} \mathrm{~V}^{-1} \mathrm{~s}^{-1}$, corresponding to a large-angle scattering length $\ell=6.8 \mu \mathrm{m}$. Irradiation with bandgap radiation shows no persistent photoeffect, but lowers the hole density and mobility.

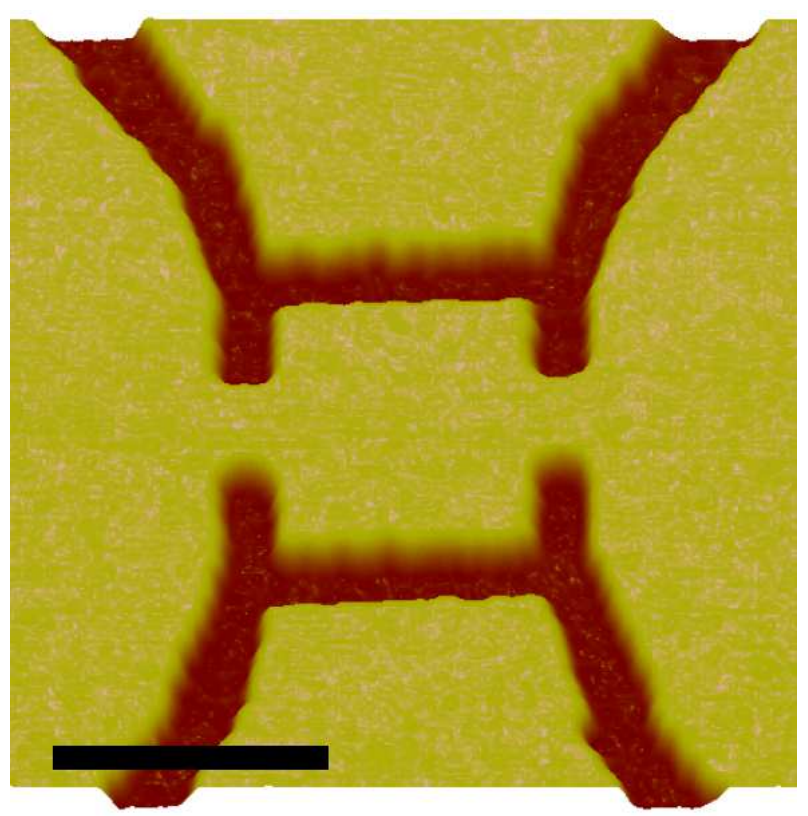

FIG. 1. (Color online) Atomic force micrograph of the etched quantum dot, prior to polyimide and gate deposition. The $120 \mathrm{~nm}$ deep trenches (red/dark) define a nominally $1 \mu \mathrm{m} \times 1 \mu \mathrm{m}$ square quantum dot connected to $2 \mathrm{DHG}$ source and drain reservoirs via two quantum point contacts (QPCs). The scale bar represents $1 \mu \mathrm{m}$.

To monitor both the state of our MBE and the doping density in the $p$-type heterostructure, an $n$-type $\mathrm{Al}_{0.34} \mathrm{Ga}_{0.66} \mathrm{As} / \mathrm{GaAs}$ heterostructure was grown on a second half of a semi-insulating 2" GaAs (100)-oriented wafer during the same MBE-growth. We obtained an electron density $n=2.8 \times 10^{11} \mathrm{~cm}^{-2}$ and mobility $\mu=7343 \mathrm{~cm}^{2} \mathrm{~V}^{-1} \mathrm{~s}^{-1}$ at room temperature. In dividing this electron density $n$ by the thickness of the Si-doped $\mathrm{Al}_{0.34} \mathrm{Ga}_{0.66} \mathrm{As}$ layer of $80 \mathrm{~nm}$, we obtain the Si-dopant density $N_{S i}=3.5 \times 10^{16} \mathrm{~cm}^{-3}$ mentioned above, assuming a full activation of the donors and transfer of all electrons into the heterointerface at room temperature. At $T=4.2 \mathrm{~K}$, we measured an electron density $n=$ $1.87 \times 10^{11} \mathrm{~cm}^{-2}$ and mobility $\mu=1.42 \times 10^{6} \mathrm{~cm}^{2} \mathrm{~V}^{-1} \mathrm{~s}^{-1}$ before illumination and $n=5.22 \times 10^{11} \mathrm{~cm}^{-2}$ with $\mu=$ $2.19 \times 10^{6} \mathrm{~cm}^{2} \mathrm{~V}^{-1} \mathrm{~s}^{-1}$ after illumination with bandgap radiation (persistent photo effect). This wafer was also used to verify the expected electrical performance of devices fabricated from this material growth (see Ref. ${ }^{26}$ ).

To fabricate the hole quantum dot, a $140 \mathrm{~nm}$ high Hall bar mesa was patterned using standard photolithography and wet etching techniques. Photolithographically defined ohmic contacts were formed by thermally evaporating $150 \mathrm{~nm}$ of AuBe alloy and annealing at $490^{\circ} \mathrm{C}$ for $90 \mathrm{~s}$. The $1 \mu \mathrm{m}^{2}$ quantum dot was defined by electron beam lithography and wet etching with 1:8:259 $\mathrm{H}_{2} \mathrm{SO}_{4}: \mathrm{H}_{2} \mathrm{O}_{2}: \mathrm{H}_{2} \mathrm{O}$ solution to a trench depth of $120 \mathrm{~nm}$. Figure 1 shows an atomic force micrograph of the resulting dot structure. The dot was covered with a $140 \mathrm{~nm}$ 


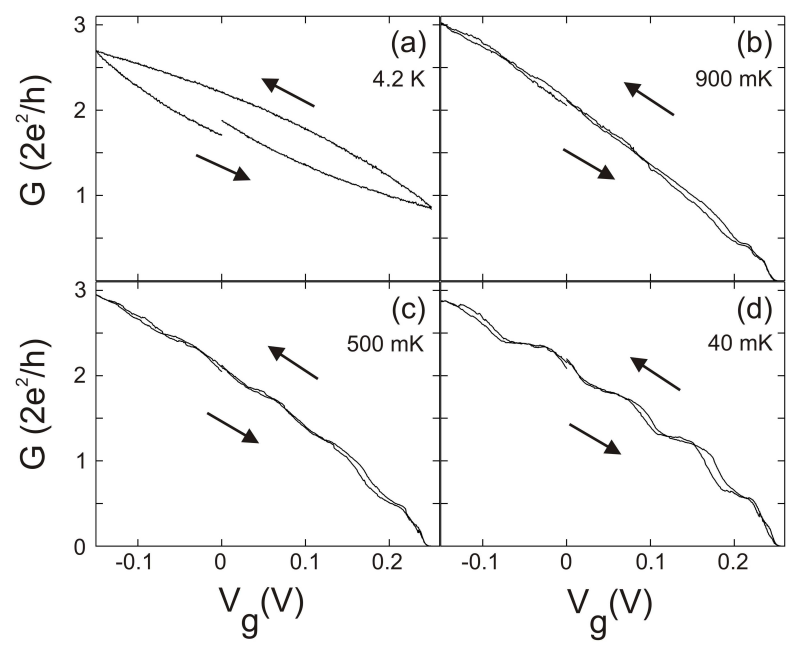

FIG. 2. Conductance $G$ at $B=0$ vs gate voltage $V_{g}$ at (a) $T=4.2 \mathrm{~K}$ (b) $900 \mathrm{mK}$ (c) $500 \mathrm{mK}$ and (d) $40 \mathrm{mK}$ at $V_{g}$ sweep rate of $1 \mathrm{mV} / \mathrm{s}$. The hysteresis reduces with $T$ as charge migration between acceptor layer trap sites gradually freezes out. Conductance plateaus also emerge with reduced $T$, generated by the dot's entrance and exit QPCs.

thick polyimide insulating layer and an evaporationdeposited $20 \mathrm{~nm} \mathrm{Ti} / 80 \mathrm{~nm} \mathrm{Au}$ top gate. A top gate voltage $V_{g}$ modulates the hole density $p$ in the dot and surrounding $2 \mathrm{DHG}$, and also alters the dot's remote ionized dopant potential, as discussed below. The dot's electrical conductance $G$ was measured using standard four-terminal lock-in techniques with a $100 \mu \mathrm{V}$ excitation voltage at $13 \mathrm{~Hz}$. The current $I_{s d}$ at $B=0 \mathrm{~T}$ ranged between $1-15 \mathrm{nA}$ depending on the value of $V_{g}$. A Keithley 2400 was used to control $V_{g}$ enabling continuous gate leakage current monitoring; this current was $<0.5 \mathrm{nA}$ throughout the experiment. The device was mounted on a cold finger thermally linked to the mixing chamber of an Oxford Instruments Kelvinox K100 dilution refrigerator enabling measurements in the temperature range $40 \mathrm{mK}<T<0.9 \mathrm{~K}$, with two additional points at discrete temperatures of $4.2 \mathrm{~K}$ and $300 \mathrm{~K}$. The device was located inside a superconducting solenoid, enabling a variable magnetic field $|B|<10 \mathrm{~T}$ to be applied perpendicular to the 2DHG plane.

Our analysis relies on subtractive methods to compare sets of MCF traces. Thus we were careful to ensure each MCF trace was obtained under experimental conditions that were as identical as possible. This included ensuring that all MCF traces were obtained at fixed excitation voltage with the mixing chamber temperature $T=40 \mathrm{mK}$, and that MCF traces were only compared if they have the same $G(B=0)$ to within $0.05 \times 2 \mathrm{e}^{2} / \mathrm{h}$ since the dot's hole density also influences the MCF. Additionally, we deliberately maintain a relatively high excitation voltage to maximise the signal-to-noise ratio of our MCF traces. Together these allow us to attribute changes in MCF solely to changes in acceptor configuration.

\section{RESULTS}

\section{A. Temperature dependence of gate characteristics}

Figure 2 shows how the conductance at zero magnetic field $G(B=0)$ versus $V_{g}$ evolves as $T$ is reduced. Significant hysteresis is observed at $T=4.2 \mathrm{~K}$ as $V_{g}$ is first swept to $V_{g}>0$ (depletion) and then $V_{g}<0$ (accumulation). The anticlockwise hysteresis loop is consistent with Ref. ${ }^{26}$, indicating transport is influenced by gateinduced charge transfer between trap sites located between the gate and 2DHG. The hysteresis reduces as $T$ is reduced (Figs. 2(b-d)) due to a reduction in the available thermal energy $k T$ relative to the trap energy $E_{A}$. Conductance quantization develops with reduced $T$; the plateaus sit at non-integer multiples of $G_{0}=2 e^{2} / h$ due to the non-additivity of QPCs in series separated by less than $\ell \underline{\underline{31,32}}$

A noteworthy aspect is that the charge migration/hysteresis means our devices' electrical characteristics evolve with both time $t$ and experimental parameter history. Thus Fig. 2 should be considered only as a 'snapshot' of the device at that time. In particular, readers should be cautious in using Fig. 2(d) to map $G$ to $V_{g}$ for Figures 3-5, as the $G(B=0)$ versus $V_{g}$ characteristic offsets horizontally depending on device parameter history. To deal with this, we returned to particular $G(B=0)$ values where quantized conductance plateaus occur to compare $\mathrm{MCF}$, rather than comparing $\mathrm{MCF}$ at a specific $V_{g}$. Simply returning to a given $V_{g}$ results in much larger changes in $G(B=0)$ and the MCF than in Figures 3-5.

\section{B. Temporal stability of the MCF}

The hysteresis in Fig. 2(d) indicates some charge between the gate and 2DHG remains mobile even at $T=40 \mathrm{mK}$. The first question, by necessity, is the timescale over which the charge configuration remains stable at fixed $V_{g}$ and minimum $T$. This is essential to knowing whether $G(B)$ provides a meaningful quasistatic magnetofingerprint of transport on the timescale for obtaining a $G(B)$ trace, typically $\sim 20 \mathrm{~min}$, and that $G(B)$ obeys known behaviors e.g., the Onsager-Casimir symmetry relation $G(B)=G(-B) \stackrel{33,34}{3}$ Note that the defined dot geometry is fixed, so MCF changes at fixed $G(B=0)$ directly reflect changes in the dot's underlying disorder potential. 13,14

Figures $3(\mathrm{a} / \mathrm{b})$ show how $G(B)$ evolves at fixed $V_{g}$, with traces at times $t=0$ (black), 8 (blue) and 15 hours (green) after setting $V_{g}=-58.5 \mathrm{mV}$ and $-156 \mathrm{mV}$ to give (a) $G(B=0) \sim 1.8 G_{0}$ and (b) $G(B=0) \sim$ $2.5 G_{0}$, respectively. We obtain almost identical MCF at $t=0,8$ and 15 hrs with $V_{g}=-58.5 \mathrm{mV}$ (Fig. 3(a)), but MCF evolves markedly over time with $V_{g}=-156 \mathrm{mV}$ (Fig. 3(b)), indicative of significant disorder potential changes within the dot $\stackrel{13,14}{14}$ To highlight this, Figures $3(\mathrm{c} / \mathrm{d})$ present the conductance difference $\Delta(B)=$ 

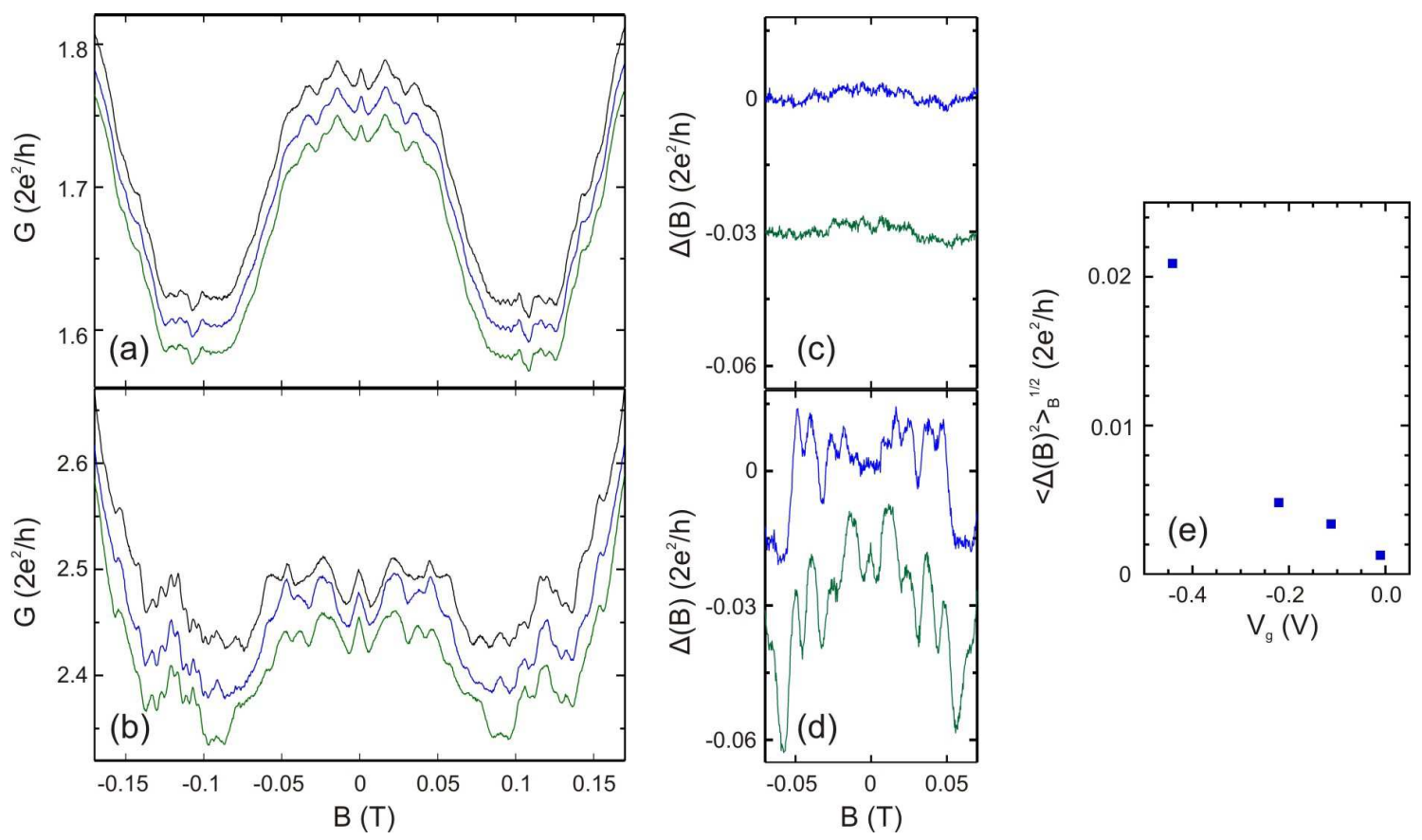

FIG. 3. (Color online) Magnetoconductance $G(B)$ with $G(B=0)$ set to the plateaus at (a) 1.8 and (b) $2.5 G_{0}$, using $V_{g}=-58.5$ and $-156 \mathrm{mV}$, respectively. In each case three traces are obtained at times $t=0$ (black), 8 (blue) and 15 hours (green) after setting $V_{g}$. Traces offset vertically for clarity by (from top) 0,0 and $-0.02 G_{0}$ in (a) and $0,-0.04$ and $-0.07 G_{0}$ in (b). The background $G$ suffers a small drift between traces (accounted for in vertical offsets) due to changes in trapped charge in the 2DHG source and drain reservoirs (see text). (c) and (d) show the corresponding conductance difference $\Delta(B)=\delta G(B)-\langle\delta G(B)\rangle_{B}$ between the $t=8$ and 0 hour traces (blue) and $t=15$ and 0 hour traces (green) vs $B$. Each green trace is offset by $-0.03 G_{0}$ (e) root-mean-square conductance difference $\left\langle\Delta(B)^{2}\right\rangle_{B}^{\frac{1}{2}}$ vs $V_{g}$ for pairs of $G(B)$ traces separated by a period of $1 \mathrm{hr}$ at fixed $V_{g}$ showing the increased MCF changes as the gate electric field shifts the device further from its equilibrium acceptor charge configuration at cooldown (i.e., $V_{g}=0$ ).

$\delta G(B)-\langle\delta G(B)\rangle_{B}$ where $\delta G(B)=G(B, t)-G(B, 0)$ for $t=8$ or 15 hours. The subtraction of $\langle\delta G(B)\rangle_{B}$ removes any background d.c. conductance offset due to changes in trapped charge outside the dot/QPCs and more than a coherence length away. This offset is not always gradual; random 'jumps' to higher/lower $G$ occur every few hours. One example occurred between the top and middle traces in Fig. 3(a), removing the need for a vertical offset for the blue trace. Thus, changes in $G(B)$ show up in $\Delta(B)$ as fluctuations around $\Delta(B)=0$.

Fig. 3(c) shows that $\Delta(B)$ remains close to 0 for all $B$ with $V_{g}=-58.5 \mathrm{mV}$, as expected for almost identical $G(B)$ traces. Conversely, substantial $\Delta(B)$ fluctuations emerge for $V_{g}=-156 \mathrm{mV}$ (Fig. 3(d)), with the magnitude of the fluctuations increasing with time. The marked changes to disorder potential at more negative $V_{g}$ suggests the gate electric field drives this underlying change in disorder potential. One possible scenario is: cooling the device at $V_{g}=0$ sets an initial equilibrium state for trapped charge in the Si acceptor layer. The equilibrium bandstructure in this finite thickness layer is such that the occupied trap density in the growth direction $z$ is inhomogeneous; this picture is consistent with observations for the deep-trapping Si DX center in (100)oriented AlGaAs/GaAs heterostructures, $\stackrel{16,29}{\longleftarrow}$ albeit with shallower traps here. A non-zero $V_{g}$ produces an electric field along $z$ that drives charge migration between trap sites. If $k T<E_{A}$ then the migration rate is slow and should increase with the difference between $V_{g}$ and its value during cool-down (i.e., $V_{g}=0$ ).

To test this scenario, we took pairs of $G(B)$ traces separated by $1 \mathrm{hr}$, starting at $V_{g}=0 \mathrm{~V}$ and using progressively more negative $V_{g}$. To quantify MCF differences, i.e., the magnitude of $\Delta(B)$ fluctuations, we plot the root-mean-square conductance difference $\left\langle\Delta(B)^{2}\right\rangle_{B}^{\frac{1}{2}}$ versus $V_{g}$ in Figure $3(\mathrm{e})$. The quantity $\left\langle\Delta(B)^{2}\right\rangle_{B}^{\frac{1}{2}}$ is similar to the correlation $F$ used previously $, 13,14,35$ and differs only by the absence of a normalization coefficient based on random traces $\stackrel{35}{ }$ For completeness, corresponding $F$ values are shown in the Appendix. Fig. 3(e) shows a clear increase in $\left\langle\Delta(B)^{2}\right\rangle_{B}^{\frac{1}{2}}$ with more negative $V_{g}$, consistent with gate field driven charge migration in the $\mathrm{Si}$ acceptor layer.

\section{Evolution of MCF with gate modulation}

Figure 3(e) naturally leads to the question: How robust is the MCF if $V_{g}$ is swept to some distant value 

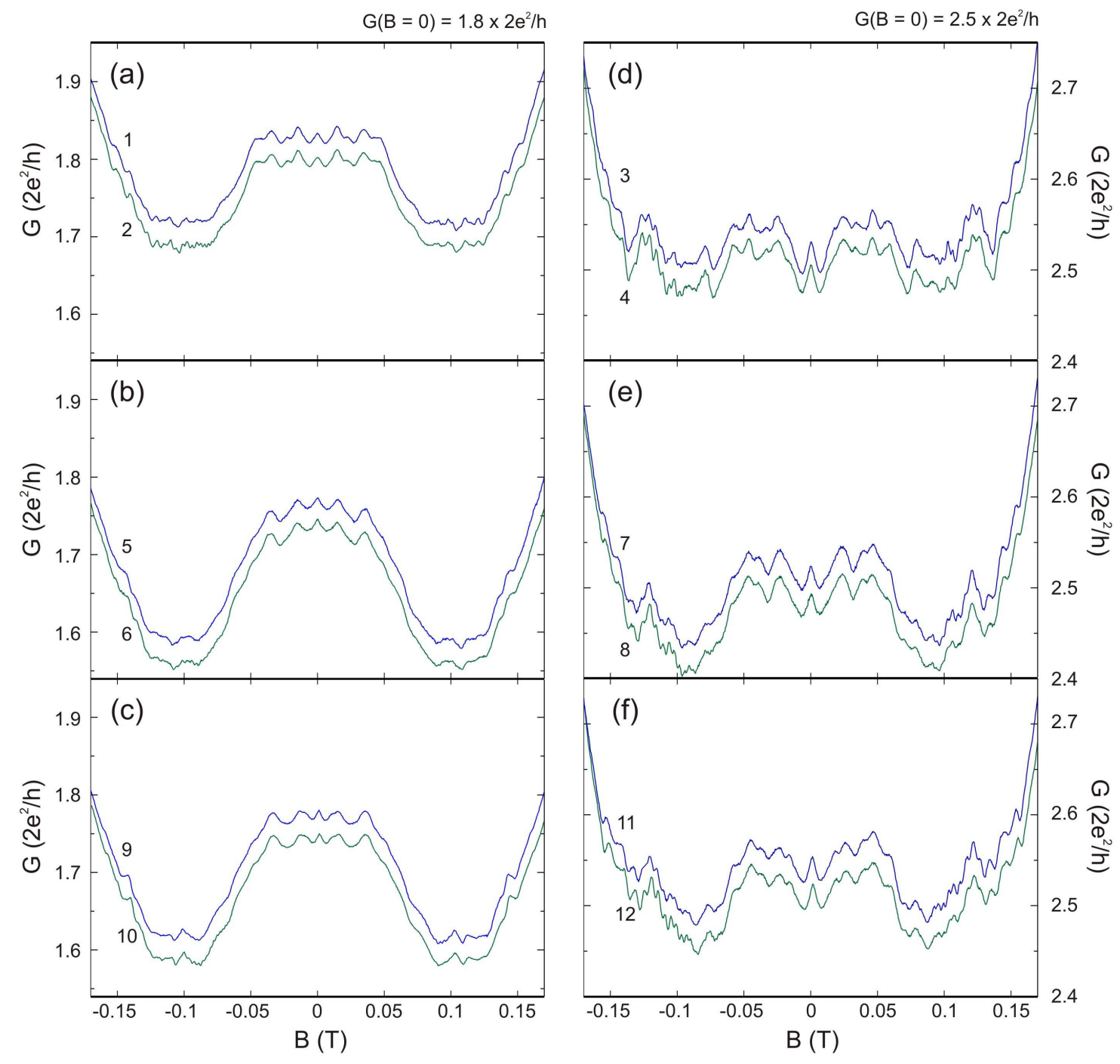

FIG. 4. (Color online) MCF traces obtained at (a-c) $G(B=0)=1.8 G_{0}$ and (d-f) $G(B=0)=2.5 G_{0}$ with the numbers $1-12$ indicating the order the traces were obtained in. The protocol is to: Set $G(B=0)=1.8 G_{0}$, obtain $G(B)$ [trace 1 in (a)], wait $1 \mathrm{hr}$, obtain $G(B)$ [trace 2 in (a)], set $G(B=0)=2.5 G_{0}$, obtain $G(B)$ [trace 3 in (d)], wait $1 \mathrm{hr}$, obtain $G(B)$ [trace 4 in (d)], set $G(B=0)=1.8 G_{0}$, obtain $G(B)$ [trace 5 in (b)], wait $1 \mathrm{hr}$, obtain $G(B)$ [trace 6 in (b)], and so on through the remaining 6 traces. The second (green) trace in each panel is offset vertically for clarity by $-0.03 G_{0}$.

and returned to its original setting? In other words, to what extent does charge migration in $z$ produce changes in charge distribution in the $x-y$ plane, i.e., ionized dopant potential? This will be linked to trap site population - if traps are mostly filled, charge may migrate in $z$ and ultimately return to the same initial trap site under a cyclic variation in $V_{g}$; if they are mostly empty, the probability of return will be low.

Figure 4 shows pairs of $G(B)$ traces obtained alternately at the $G(B=0)=1.8$ and $2.5 G_{0}$ plateaus. Two traces were taken at each gate voltage to directly compare the temporal stability to disorder induced by the gate potential over a similar time scale: The numbers $1-12$ in Fig. 4 indicate the sequence in which traces were obtained. The left (right) column in Fig. 4 should be con- sidered as a cyclic variation from $G=1.8 G_{0}\left(2.5 G_{0}\right)$ to $G=2.5 G_{0}\left(1.8 G_{0}\right)$ and back; as such we do this experiment twice, once starting at more positive $V_{g}$ and cycling to more negative $V_{g}$ (left column), and once vice versa (right column). If cyclic variation produced no change in the dot's disorder potential, we would expect six identical $G(B)$ traces at $G(B=0)=1.8 G_{0}$, and a different set of six identical $G(B)$ traces at $G(B=0)=2.5 G_{0}$; the difference in $G(B)$ between the $G=1.8$ and $2.5 G_{0}$ plateaus is caused by the underlying difference in hole density. Inspection of Fig. 4 makes it clear the disorder potential changes with cyclic variation in $V_{g}$. Specifically, $G(B)$ varies more between panels than within each panel, suggesting that cyclic variation in $V_{g}$ produces stronger $G(B)$ changes than background temporal changes. 
To confirm this, we again used $\left\langle\Delta(B)^{2}\right\rangle_{B}^{\frac{1}{2}}$ to quantify the $G(B)$ changes: Pairs of traces in each panel give an average $\left\langle\Delta(B)^{2}\right\rangle_{B}^{\frac{1}{2}}$ of $1.1 \times 10^{-3} G_{0}\left(2.6 \times 10^{-3} G_{0}\right)$ for the left (right) column of Fig. 4. In contrast, if we compare the second traces in a/b, b/c and a/c (or d/e, e/f and d/f) we get an average $\left\langle\Delta(B)^{2}\right\rangle_{B}^{\frac{1}{2}}$ of $10.3 \times 10^{-3} G_{0}$ $\left(9.7 \times 10^{-3} G_{0}\right)$. The $\left\langle\Delta(B)^{2}\right\rangle_{B}^{\frac{1}{2}}$ values relating to cyclic variation are $5-10 \times$ larger than those relating to temporal changes: this confirms that significant changes in dot disorder potential result from cyclic variation in $V_{g}$. The fact that the MCF continues to evolve through the 6 cycles also suggests that the trap site population is low in this sample. This is not surprising, as the primary purpose of the dopant charge is to populate the $2 \mathrm{DHG}$ and quantum dot.

\section{Evolution of MCF with thermal cycling}

The previous two sections showed that at $T=40 \mathrm{mK}$, charge migration occurs between some proportion of $\mathrm{Si}$ acceptors in the AlGaAs layer under the influence of an electric field, but spontaneous re-organization does not occur. This suggests a metastable configuration: i.e., the thermal energy $k T$ at $40 \mathrm{mK}$ is slightly lower than the activation energy $E_{A}$ for the $\mathrm{Si}$ acceptors. We thus attempted to determine $E_{A}$ via temperature studies of $G(B)$. The probability of trap deoccupation depends on $k T$ relative to $E_{A}$, thus elevating $T$ should lead to more rapid changes in disorder potential and thereby in $G(B)$, reflected by increased $\left\langle\Delta(B)^{2}\right\rangle_{B}^{\frac{1}{2}}$. Scannell et al. ${ }^{14}$ recently performed such a study for GaAs and InGaAs electron quantum dots using the following methodology: Set and hold a particular $V_{g}$, obtain $G(B)$ at base temperature $T_{0}=300 \mathrm{mK}$, heat to $T_{1}>>300 \mathrm{mK}$ for some time $t$, return to $T_{0}$ and remeasure $G(B)$, heat to $T_{2}>T_{1}$ for $t$, return to $T_{0}$ and remeasure $G(B)$, repeat $i$ times until $T_{i}=300 \mathrm{~K}$. The $G(B)$ at each iteration $i$ is then compared to the initial $G(B)$ trace, with the correlation $F=1-\left\langle\Delta(B)^{2}\right\rangle_{B}^{\frac{1}{2}} / N$, where $N$ is a normalization factor, plotted against $T_{i}$ to identify where $k T_{i}$ becomes sufficient to cause major changes to $G(B) \underline{\underline{14}}$

Repeating the study by Scannell et al. for a hole quantum dot, as we do here, faces two serious challenges. The higher effective mass for holes results in a significantly reduced phase coherence time relative to electrons $\frac{36}{\underline{6}}$ As a result $G(B)$ must be measured at lower $T$ to obtain MCF; the MCF of our device is quenched at $T \gtrsim 100 \mathrm{mK}$. This means our measurements require a dilution refrigerator, where the available $T_{i}$ range is limited to $T_{i}<900 \mathrm{mK}$, $T_{i}=4.2 \mathrm{~K}$ and $T_{i}=300 \mathrm{~K}$. In contrast, the ${ }^{3} \mathrm{He}$ system used in Ref $\frac{14}{\underline{14}}$ allows continuous, precise variation of $T_{i}$ over the range $300 \mathrm{mK}<T_{i}<200 \mathrm{~K}$. The second challenge is that $E_{A}$ is much smaller for our system. This means that even if we had good control over $T_{i}$ above $900 \mathrm{mK}$, much of this range is of limited use as we should reach the point where $\left\langle\Delta(B)^{2}\right\rangle_{B}^{\frac{1}{2}}$ saturates at its maximum value at relatively low $T_{i}$. Nonetheless, we attempt the study within these limitations to confirm at least that $E_{A}$ for $\mathrm{Si}$ acceptors in (311)A AlGaAs/GaAs heterostructures is much smaller than for $\mathrm{Si}$ donors in (100) AlGaAs/GaAs heterostructures.

Figure 5 shows $G(B)$ traces obtained before and after cycling to $T_{i}=0.3,0.5,0.7,0.9$ and $4.2 \mathrm{~K}$. We first make one important note regarding interpretation relative to Ref $\stackrel{14}{*}$; here we compare the $G(B)$ traces obtained immediately before/after cycling to $T_{i}$, rather than comparing $G(B)$ after to a common, initial ' $T_{0}$ ' $G(B)$ trace. This is to more fairly deal with the continuous temporal evolution/relaxation in $G(B)$; i.e., we do not want our analysis to include the temporal changes in $G(B)$ observed in Fig. 3. The MCF in Fig. 5(a) shows little change after thermal cycling to $T_{i}=300 \mathrm{mK}$. For this pair of traces we obtain a correlation $F=0.97$. As we increase $T_{i}$ in steps of $200 \mathrm{mK}$ in Fig. 5(b-d), the differences between MCF traces before and after cycling to $T_{i}$ begin to increase, as indicated by the arrows. Correspondingly, there is a gradual fall-off in $F$ towards $\sim 0.93$, evident in Fig. 5(f) where we plot $F$ versus $T_{i}$. There is a little scatter in $F$ for $T_{i}<900 \mathrm{mK}$, but this is also apparent in Ref $\stackrel{14}{\underline{14}}$, and often results from small, slow background variations running through the data. Ultimately, upon reaching $T_{i}=900 \mathrm{mK}, F$ has not dropped off markedly.

While the data in Fig. 5(a-d) could be obtained at fixed $V_{g}$, with $T$ controllably increased to $T_{i}$, held there for $30 \mathrm{~min}$ and then returned to $T=40 \mathrm{mK}$ for $G(B)$ measurement, this is not possible for the next available $T_{i}=4.2 \mathrm{~K}$. More effort is needed to reach $4.2 \mathrm{~K}$ with a dilution refrigerator, i.e., remove of ${ }^{3} \mathrm{He} /{ }^{4} \mathrm{He}$ mixture, add exchange gas, equilibrate with $4.2 \mathrm{~K}$ bath, pump out exchange gas, recondense mixture and restart circulation; this process takes many hours. Additionally, the resulting dopant reorganization inevitably means that $G \sim 2.5 G_{0}$ does not occur at the same $V_{g}$. Indeed, the initial and final $G(B)$ traces in Fig. 5(e) were obtained at $V_{g}=-0.156$ and $-0.270 \mathrm{~V}$, respectively. They are markedly different, and the corresponding $F=0.14$.

Fig. 5(f) shows that the $T_{i}$ where the MCF decorrelates rapidly lies somewhere in the range $0.9<T_{i}<4.2 \mathrm{~K}$. The rapid $F$ drop-off for an AlGaAs/GaAs electron dot in Ref $\frac{14}{-14}$ occurs at $T_{i}=165 \mathrm{~K}$. If we estimate that $T_{i} \sim 2 \mathrm{~K}$ for $F=0.5$ in Fig. 5(f), then we would expect the trap depth for our Si acceptors to be $1.2 \%$ of that for Si donors. Assuming the Si donors are DX centers with depth $\sim 250 \mathrm{meV}, \stackrel{28}{\stackrel{2}{2}}$ then we can estimate the shallow Si acceptor trap depth for our dots to be $E_{A}=3 \mathrm{meV}$. The isolated $\mathrm{Si}$ acceptor energy in $\mathrm{GaAs}$ is $34.8 \mathrm{meV}^{37}$ and $\sim 60 \mathrm{meV}$ in $\mathrm{AlGaAs}{ }^{38}$; our trap $E_{A}$ is over an order of magnitude smaller. This value of $E_{A}=3 \mathrm{meV}$ should be considered a lower bound estimate as the difficulties involved in obtaining the $T_{i}=4.2 \mathrm{~K}$ measurement could have reduced the measured $F$. However, even if the decorrelation temperature were as high as $T=20 \mathrm{~K}$, the corresponding $E_{A}=30 \mathrm{meV}$ would still be $\sim 2 \times$ smaller 

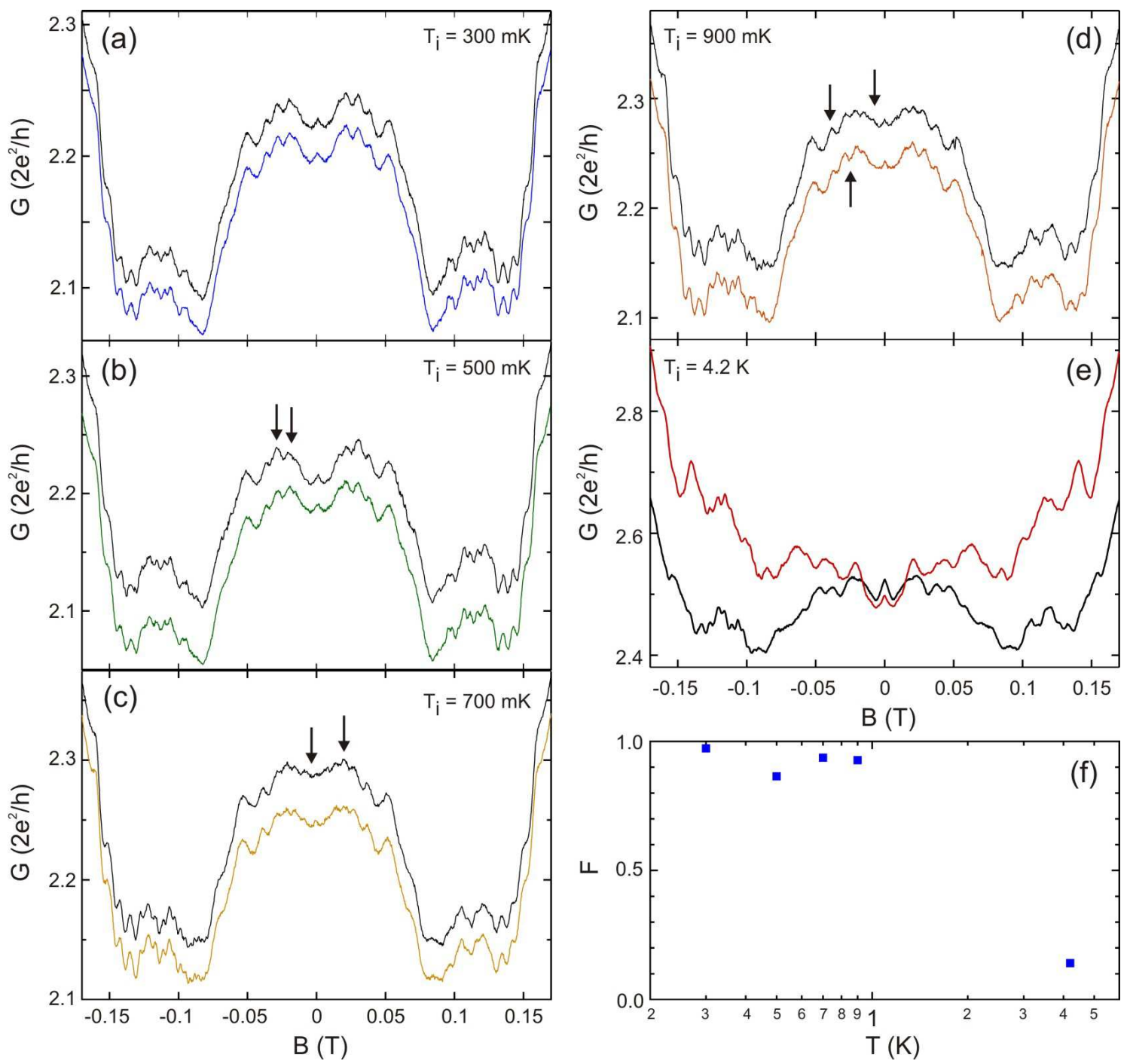

FIG. 5. (Color online) MCF traces before and after (upper and lower trace in each frame, respectively) cycling to intermediate temperatures $\left(T_{i}\right)$ of (a) $300 \mathrm{mK}$ (b) $500 \mathrm{mK}$ (c) $700 \mathrm{mK}$ (d) $900 \mathrm{mK}$ and (e) $4.2 \mathrm{~K}$. Traces in (a - d) were obtained with $V_{g}=0$, and $T_{i}$ was held for 30 mins. For (e), $V_{g}=-0.156$ and $-0.270 \mathrm{~V}$ for the initial and final traces, respectively, and the temperature variation was more complex, as discussed in the text. The lower traces in (a), (b) and (c) have a vertical offset of $-0.01 G_{0},-0.04 G_{0}$ and $-0.03 G_{0}$, respectively. The differences in $G(B=0)$ between traces in each experiment were (a) $-0.017 G_{0}$, (b) $0.015 G_{0}$, (c) $0.007 G_{0}$, (d) $-0.037 G_{0}$ and (e) $0.03 G_{0}$. Cycling to $300 \mathrm{mK}$ produces no change in the MCF, but changes emerge upon cycling to $\geqslant 500 \mathrm{mK}$. (f) The correlation $F$ vs $T_{i}$ for pairs of $G(B)$ traces in (a - e).

than the isolated Si-acceptor level in AlGaAs. One possible source of this small trap is the Si-X acceptor defect, $\underline{39}$ which appears at very high Si doping densities. As Ashwin et al. point out, Si-X would need a small activation energy to be electrically active amongst a high density of isolated $\mathrm{Si}$ acceptors $\underline{\underline{40}}$

\section{CONCLUSIONS}

We have used the sensitivity of the magnetoconductance fluctuations in an open quantum dot to small-angle scattering from remote ionized impurities ${ }^{13,14}$ to study the stability of Si acceptors in (311)A AlGaAs/GaAs het- erostructures. With $T=40 \mathrm{mK}$ and $V_{g} \sim 0$, the $\mathrm{MCF}$ remain reproducible for a period $\sim 15 \mathrm{hrs,} \mathrm{demonstrat-}$ ing that under the simplest quasi-static conditions, a hole quantum dot can show stable, meaningful electrical characteristics and MCF, consistent with previous studies $\underline{36}$ By contrast, the temporal stability of the MCF is significantly reduced as $V_{g}$ is made more negative. Similarly, MCF changes are observed when cycling $V_{g}$ away from some initial value, and returning to a value that gives the same $G(B=0)$. We attribute this to migration of charge between trap sites in the $\mathrm{Si}$ acceptor layer due to the electric field induced between the gate and 2DHG by $V_{g}$. These results suggest a metastable state for $\mathrm{Si}$ acceptors in AlGaAs at $T=40 \mathrm{mK}$, with the 
activation energy $E_{A}$ of acceptors larger than the thermal energy $k T$. To determine $E_{A}$, we followed Scannell et al. and looked at how periods at higher temperatures $T_{i}$ changed the MCF at fixed $V_{g}$. This experiment faced many difficulties, but we found that a $T_{i}=4.2 \mathrm{~K}$ is sufficient to produce complete decorrelation of the MCF. By comparing with the known trap energy for Si DX centers and the $T_{i}$ required to produce decorrelation in electron devices, we estimate that the shallow acceptor traps in our heterostructures have an activation energy $E_{A} \sim 3 \mathrm{meV}$, consistent with our earlier studies of device hysteresis $\stackrel{26}{2}$ This work demonstrates the capacity for the MCF in quantum dots to be used as a tool for detecting temporal changes in ionized dopant configuration and charge trap occupation in semiconductor heterostructures. One structure of immediate interest is Cdoped $p$-type AlGaAs/GaAs heterostructures, since devices fabricated from this material also exhibit gate instability at low temperature $\stackrel{41}{ }$ Repeating the experiments conducted here for this material could help to determine whether charge migration between $\mathrm{C}$ acceptors plays a role in this instability, and determine the $\mathrm{C}$ acceptor activation energy in AlGaAs.

\section{ACKNOWLEDGMENTS}

This work was funded by Australian Research Council Grants DP0110103802, FT0990285, DP120101859, DP120102888 and by the Australian Government under the Australia-India Strategic Research Fund. DR and ADW acknowledge support from DFG SPP1285 and BMBF QuaHL-Rep 16BQ1035. This work was performed in part using the NSW node of the Australian National Fabrication Facility (ANFF). We thank L. Eaves for helpful discussions on donors and acceptors in $\mathrm{Al}$ GaAs/GaAs heterostructures.

\section{Appendix: Correlation data for Figure 3(e)}

Figure 6 shows the correlation $F$ versus gate voltage $V_{g}$ corresponding to the data presented in Figure 3(e). The correlation $F$ is obtained by applying a cross-correlation analysis to a pair of traces $G_{1}(B)$ and $G_{2}(B)$ :

$$
\begin{aligned}
& F=\sqrt{1-\frac{\left\langle\left[G_{1}(B)-G_{2}(B)\right]\right\rangle_{B}}{N}} \quad \text { where } \\
& N=\left\langle\left[G_{x}(B)-G_{y}(B)\right]\right\rangle_{B} .
\end{aligned}
$$

The symbol \langle\rangle$_{B}$ represents an average over the 14001 data points spanning $-B_{c}<B<B_{c}$, where $B_{c}=0.07 \mathrm{~T}$ is the field at which the cyclotron diameter matches the billiard width. The normalization constant $N$ is calculated by averaging the correlations of 100 pairs of randomly generated $G(B)$ traces with the same average, maximum and minimum $G$ as the two analysed traces

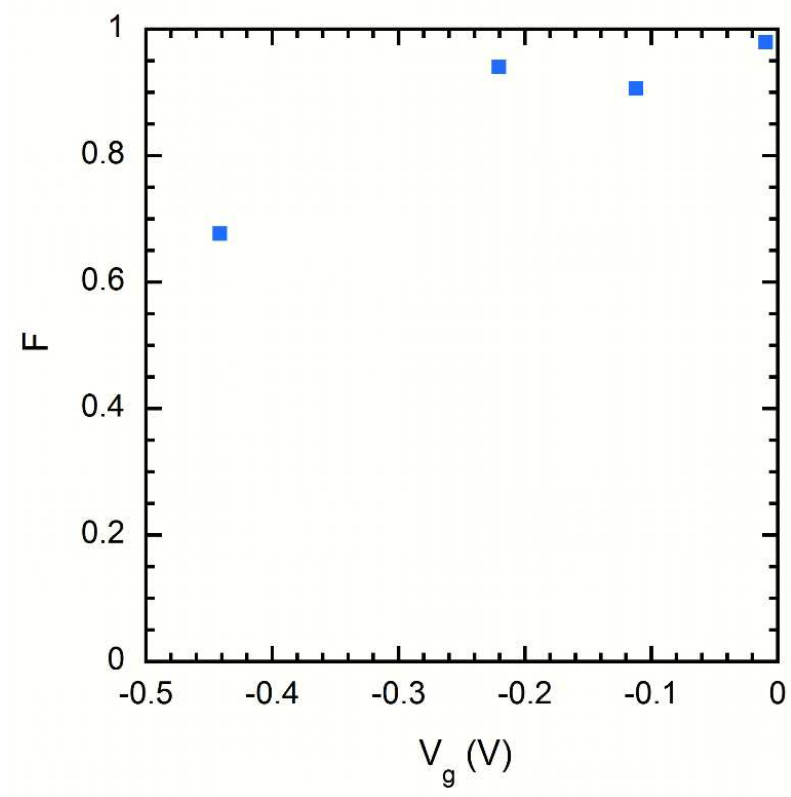

FIG. 6. Correlation $F$ vs gate voltage $V_{g}$ corresponding to the data presented in Fig. 3(e) of the main text.

$G_{1}(B)$ and $G_{2}(B)$. Adopting this normalization, the correlation scale varies between 1 for mathematically identical traces to 0 for complete decorrelation. Further details of this method can be found in Refs. ${ }^{13,14,35}$.
* adam.micolich@nanoelectronics.physics.unsw.edu.au

1 A. Y. Cho, Appl. Phys. Lett. 19, 467 (1971).

2 R. Dingle, H. L. Stormer, A. C. Gossard, and W. Wiegmann, Applied Physics Letters 33, 665 (1978).

3 M. A. Kastner, Phys. Today 46, 24 (1993).

${ }^{4}$ L. P. Kouwenhoven, D. G. Austing, and S. Tarucha, Rep. Prog. Phys. 64, 701 (2001).

${ }^{5}$ R. Hanson, L. P. Kouwenhoven, J. R. Petta, S. Tarucha, and L. M. K. Vandersypen, Rev. Mod. Phys. 79, 1217 (2007).

6 T. D. Ladd, F. Jelezko, R. Laflamme, Y. Nakamura,
C. Monroe, and J. L. O'Brien, Nature 464, 45 (2010).

7 J. J. Lin and J. P. Bird, J. Phys.: Condens. Matter 14, R501 (2002).

8 J. P. Bird, R. Akis, D. K. Ferry, A. P. S. de Moura, Y.C. Lai, and K. M. Indlekofer, Rep. Prog. Phys. 66, 583 (2003).

9 A. P. Micolich, A. M. See, B. C. Scannell, C. A. Marlow, T. P. Martin, I. Pilgrim, A. R. Hamilton, H. Linke, and R. P. Taylor, Fortschritte der Physik 61, 332 (2013).

10 Y. Aharonov and D. Bohm, Phys. Rev. 115, 485 (1959).

11 C. M. Marcus, A. J. Rimberg, R. M. Westervelt, P. F. 
Hopkins, and A. C. Gossard, Phys. Rev. Lett. 69, 506 (1992).

12 S. Feng, P. A. Lee, and A. D. Stone, Phys. Rev. Lett. 56, 1960 (1986).

13 A. M. See, I. Pilgrim, B. C. Scannell, R. D. Montgomery, O. Klochan, A. M. Burke, M. Aagesen, P. E. Lindelof, I. Farrer, D. A. Ritchie, R. P. Taylor, A. R. Hamilton, and A. P. Micolich, Phys. Rev. Lett. 108, 196807 (2012).

14 B. C. Scannell, I. Pilgrim, A. M. See, R. D. Montgomery, P. K. Morse, M. S. Fairbanks, C. A. Marlow, H. Linke, I. Farrer, D. A. Ritchie, A. R. Hamilton, A. P. Micolich, L. Eaves, and R. P. Taylor, Phys. Rev. B 85, 195319 (2012).

15 C. Buizert, F. H. L. Koppens, M. Pioro-Ladrière, H.-P. Tranitz, I. T. Vink, S. Tarucha, W. Wegscheider, and L. M. K. Vandersypen, Phys. Rev. Lett. 101, 226603 (2008).

16 M. Pioro-Ladrière, J. H. Davies, A. R. Long, A. S. Sachrajda, L. Gaudreau, P. Zawadzki, J. Lapointe, J. Gupta, Z. Wasilewski, and S. Studenikin, Phys. Rev. B 72, 115331 (2005).

17 J.-B. Yau, E. P. De Poortere, and M. Shayegan, Phys. Rev. Lett. 88, 146801 (2002).

18 R. Winkler, S. J. Papadakis, E. P. DePoortere, and M. Shayegan, Phys. Rev. Lett 85, 4574 (2000).

19 R. Danneau, O. Klochan, W. R. Clarke, L. H. Ho, A. P. Micolich, M. Y. Simmons, A. R. Hamilton, M. Pepper, D. A. Ritchie, and U. Zülicke, Phys. Rev. Lett. 97, 026403 (2006).

20 J. C. H. Chen, O. Klochan, A. P. Micolich, A. R. Hamilton, T. P. Martin, L. H. Ho, U. Zülicke, D. Reuter, and A. D. Wieck, New J. Phys 12, 033043 (2010).

21 O. Klochan, A. P. Micolich, A. R. Hamilton, K. Trunov, D. Reuter, and A. D. Wieck, Phys. Rev. Lett. 107, 076805 (2011).

22 A. Srinivasan, L. A. Yeoh, O. Klochan, T. P. Martin, J. C. H. Chen, A. P. Micolich, A. R. Hamilton, D. Reuter, and A. D. Wieck, Nano Lett. 13, 148 (2013).

${ }^{23}$ I. Zailer, J. E. F. Frost, C. J. B. Ford, M. Pepper, M. Y. Simmons, D. A. Ritchie, J. T. Nicholls, and G. A. C. Jones, Phys. Rev. B 49, 5101 (1994).

24 A. J. Daneshvar, C. J. B. Ford, A. R. Hamilton, M. Y. Simmons, M. Pepper, and D. A. Ritchie, Phys. Rev. B
55, 13409 (1997).

25 L. P. Rokhinson, D. C. Tsui, L. N. Pfeiffer, and K. W. West, Superlatt. Microstruct. 32, 99 (2002).

26 A. M. Burke, D. E. J. Waddington, D. J. Carrad, R. W. Lyttleton, H. H. Tan, P. J. Reece, O. Klochan, A. R. Hamilton, A. Rai, D. Reuter, A. D. Wieck, and A. P. Micolich, Phys. Rev. B 86, 165309 (2012).

27 D. J. Carrad, A. M. Burke, P. J. Reece, R. W. Lyttleton, D. E. J. Waddington, A. Rai, D. Reuter, A. D. Wieck, and A. P. Micolich, J. Phys.: Condens. Matter 25, 325304 (2013).

28 P. M. Mooney, J. Appl. Phys 67, R1 (1990).

29 E. Buks, M. Heiblum, Y. Levinson, and H. Shtrikman, Semicond. Sci. Technol. 9, 2031 (1994).

30 W. I. Wang, E. E. Mendez, T. S. Kuan, and L. Esaki, Applied Physics Letters 47 (1985).

31 C. W. J. Beenakker and H. van Houten, Phys. Rev. B 39, 10445 (1989).

${ }^{32}$ L. P. Kouwenhoven, B. J. van Wees, W. Kool, C. J. P. M. Harmans, A. A. M. Staring, and C. T. Foxon, Phys. Rev. B 40, 8083 (1989).

33 L. Onsager, Phys. Rev. 38, 2265 (1931).

34 H. B. G. Casimir, Rev. Mod. Phys. 17, 343 (1945).

35 R. P. Taylor, A. P. Micolich, R. Newbury, and T. M. Fromhold, Phys. Rev. B 56, R12733 (1997).

36 S. Faniel, B. Hackens, A. Vlad, L. Moldovan, C. Gustin, B. Habib, S. Melinte, M. Shayegan, and V. Bayot, Phys. Rev. B 75, 193310 (2007).

37 R. F. Kirkman, R. A. Stradling, and P. J. Lin-Chung, J. Phys. C 11, 419 (1978).

38 N. Galbiati, E. Grilli, M. Guzzi, L. Brusaferri, L. Pavesi, and M. Henini, Semiconductor Science and Technology 11, 1830 (1996).

39 R. Murray, R. C. Newman, M. J. L. Sangster, R. B. Beall, J. J. Harris, P. J. Wright, J. Wagner, and M. Ramsteiner, J. Appl. Phys. 66, 2589 (1989).

40 M. J. Ashwin, M. R. Fahy, R. C. Newman, J. Wagner, D. A. Robbie, M. J. L. Sangster, I. Siller, E. Bauser, W. Braun, and K. Ploog, J. Appl. Phys. 76, 7839 (1994).

41 M. Csontos, Y. Komijani, I. Shorubalko, K. Ensslin, D. Reuter, and A. D. Wieck, Applied Physics Letters 97, 022110 (2010). 